\title{
Limits on quark nugget dark matter from cosmic ray detectors
}

\author{
Kyle Lawson \\ Department of Physics and Astronomy, University of British Columbia, Vancouver, BC, Canada
}

\begin{abstract}
The purpose of this talk is to highlight the potential role of large scale cosmic ray detectors in constraining the presence of certain classes of high mass dark matter candidates. These models are not easily constrained by conventional dark matter searches due to their very small flux, and thus, alternative detection techniques must be considered. I will begin with a brief review of heavy compact composite dark matter and some motivation for considering this class of models. In particular I will describe a model in which the dark matter consists of heavy "nuggets" of quarks and antiquarks, and highlight its relation to baryogenesis. As this form of dark matter is based in known physics its properties, as established by arguments from nuclear physics and electrodynamics, are strongly constrained. Based on these properties I will give a primarily qualitative description of the nuggets' interaction with visible matter and of the consequences of the passage of a dark matter nugget through the earth's atmosphere. From the general scales and properties of these events I argue that they may be detectable using cosmic ray observatories and that the largest of these observatories are likely to impose the strongest known constraints on this class of dark matter candidates.
\end{abstract}

\section{Baryogenisis and dark matter}

In order to motivate the following discussion I will begin with a brief review of the process of baryogenesis and its possible connection with the apparently unrelated phenomenon of dark matter.

The physical laws as we know them display a complete symmetry in their treatment of matter and antimatter. However, this symmetry is not manifested on cosmological scales where the contents of the universe are strongly weighted towards matter with only trace amounts of antimatter observed. The process by which this asymmetry arises is referred to as baryogenesis, and its nature remains one of the most important open problems in cosmology. It is generally assumed that the universe began in a matter/antimatter symmetric state and subsequently evolves into a state with a net positive baryon number (ie. matter abundance) as observed today. Conventionally this is thought to occur through, as yet unknown, physical processes which violate baryon number. In the following I will consider an alternative model in which, rather producing an overabundance of matter, baryogenesis occurs through the preferentially binding of antimatter in a high density state which interacts only minimally with the visible matter and is therefore not easily observable. A more detailed description of this model may be found in the original papers $[1,2]$.

In the early universe the temperature was sufficiently high that the baryonic content of the universe was in the form of a quark-gluon plasma (QGP) in which colour change is effectively screened and the individual quarks are not confined into hadrons. As the temperature fell the interaction strength between quarks increased to the point where they became confined into colour singlet baryons which make up the matter we see today. The confinement phase transition is accompanied by a chiral phase transition as it is the vacuum structure of the theory which determines its CP properties. This process may involve the formation of a network of domain walls which rapidly collapse as the QCD fields relax into their CP symmetric ground state. The interaction of these walls is strongly $\mathrm{CP}$ violating so they acquire a net baryonic (or antibaryonic) charge as they sweep through the QGP. The swept up matter is then compressed by the collapsing walls to sufficient density that it settles into a high density quark matter phase.

Through this mechanism the universe transitions from a high temperature QGP containing an equal number of quarks and antiquarks to a universe containing protons and neutrons and their antiparticles as well as nuggets of quarks and of antiquark. The baryons are too heavy to be pair produced in collisions and will rapidly annihilate as long as they continue to encounter their antiparticles. If no baryogenesis mechanism had occurred annihilations would have continue until the matter density was ten orders of magnitude lower than is observed. However, the nuggets decouple from the baryonic plasma, making them unavailable to participate in annihilations. The preferential formation of nuggets of antimatter would result in a baryonic plasma which contains an excess of matter having no antimatter partner with which to annihilate.

\section{Quark nugget dark matter}

As discussed above, a potential mechanism for baryogenesis may be the preferential confinement antiquarks into nuggets of high density quark matter. The nuggets may then serve as the dark matter. These objects should be considered "dark" not because of the fundamental weakness of their interactions, but because of their small cross-section to mass ratio. In this sense they are conceptually similar to stranglets [3], a quark matter based dark matter candidate previously proposed. This picture 
is partially motivated by the similarity in the observed matter and dark matter densities with $\Omega_{D M}=5 \Omega_{v i s}$. If the baryonic and dark matter components of the universe arise through completely unrelated physical processes, then their present day abundance could easily differ by many orders of magnitude. However, in this scenario both the visible matter and dark matter emerge through the same physical mechanism of charge separation operating at the QCD phase transition. As such, the two should be expected to acquire the same energy density up to geometric factors just as is observed. In particular the observed matter to dark matter ratio, when combined with the assumption of a universe with a zero net baryon charge, implies that the hadronic matter of the universe must be divided into visible matter, quark nuggets and antiquark nuggets in the simple ratio baryonic charges $B_{v i s}: B_{N}: \bar{B}_{N} \approx 1: 2: 3$.

The baryonic content of the nuggets is taken to be bound in a high density phase of QCD, having a density comparable to, or slightly greater than, nuclear matter. At high densities the QCD phase diagram is not well mapped either theoretically or experimentally. As the density of quark matter increases a fermi surface forms and, when the energy of particles at the fermi surface moves into the regime of asymptotic freedom, the fundamental interactions governing the system are between individual quarks rather than composite baryons. This general class of high density quark matter must exist at sufficiently large densities, though the actual structure of the different states and their relation to each other remains an open question in fundamental QCD physics. Fortunately, the observable properties of the nuggets are not strongly dependent on the exact nature of its interior. Instead most interactions can be sufficiently described based on surface physics, which may be extracted using relatively simple arguments from nuclear physics and electrodynamics.

The size distribution of the nuggets is not well constrained in the formation picture discussed above. In principle this quantity could be calculated from fundamental QCD physics, but such a calculation is not practically tractable. Instead, the mean baryon number of the nuggets will serve as the main parameter characterizing their observational properties. A variety of theoretical and observational considerations (some of which will be outlined below) suggest that the mean baryon number of the nuggets falls in the range $10^{25}<B<10^{35}$. The size and mass of the nuggets will then scale as,

$$
M \sim m_{B} B, \quad r \sim \lambda B^{1 / 3}, \quad \frac{\sigma}{M} \sim \frac{\lambda^{2}}{m_{B}} B^{-1 / 3} .
$$

Here $m_{B} \sim 1 \mathrm{GeV} / \mathrm{c}^{2}$ is the mass scale of individual baryons within the quark matter and $\lambda \sim 1 \mathrm{fm}$ is the characteristic length scale. The cross section to mass ratio $\sigma / M$ is included here as it is an important parameter in gauging the observational properties of dark matter candidates.

Physically the nuggets will be macroscopically large, with the central quark matter having a radius of at least $10^{-5} \mathrm{~cm}$. The formation of any form of quark matter favours the presence of as many different flavours of quarks as possible. Thus it will contain roughly equal numbers of each of the light quarks. However, the significantly larger mass of the strange quark ensures that they will be present in lower numbers (if at all) and that the quark matter will carry a net electrical charge. The charged quark matter core is then surrounded by a surface layer of leptons (electrons in the case of a quark nugget and positrons in the antiquark case) know as the electrosphere which ensures the nuggets' overall electrically neutrality. The electrosphere is opaque to low energy photons so that its properties determine the thermodynamics of the nugget and it is the source of most observable forms of emission.

\section{Review of current constraints}

Dark matter search techniques are generally classed as either direct or indirect. Direct searches look for a signal generated by the interaction of a passing dark matter particle with the detector while indirect searches look for consequences of dark matter interactions in other, generally astrophysical, environments. As these two search techniques involve different experimental techniques and produce distinct types of constraints I will discuss them separately.

\subsection{Indirect detection and astrophysical consequences}

Indirect detection techniques rely on the interaction between the dark matter and visible matter in a variety of astrophysical environments. Any observable consequences will thus depend on the rate of interactions along a given line of sight. In general this rate may be expressed as

$$
\Gamma=\int n_{v i s} v \sigma n_{D M} d r
$$

where $n_{v i s}$ and $n_{D M}$ are the visible and dark matter densities, $v$ is the relative velocity and $\sigma$ is the interaction cross section. The dark matter mass density is determined from its gravitational interactions, independently of the microscopic details, so that this integral generally limits the cross section to mass ratio $\sigma / M$ for various dark matter candidates. As discussed in the previous section this implies that improved observational constraints tighten the limits on the allowed baryon number as $B^{-1 / 3}$.

In this context the most important observations will come from regions, such as the galactic centre, where the overlap of the visible and dark matter distribution is largest. Consequently the galactic centre has served as an important test of any dark matter model incorporating non-gravitational interactions with visible matter. Unfortunately, the high density of visible matter also complicates the interpretation of any possible dark matter signal, as it must be extracted from the dominant contribution produced by visible astrophysical sources. At present there are no known unambiguous dark matter signals observed. There have however been a series of recent observations, across a wide range of energies, that have generated some interest in the possibility of a dark matter contribution. The possible diffuse excess emission sources include a stronger than expected $511 \mathrm{keV}$ line, broad emission in the $1-30 \mathrm{MeV}$ range, diffuse hard X-ray emission from the galactic centre, and the "WMAP haze" 
observed in the microwave. The possible contribution of quark nugget dark matter to the galactic spectrum has been analyzed in the context of these observations, and it has been found that the inclusion of a nugget contribution to the diffuse background is entirely consistent with present observations [4-8]. If quark nuggets are to provide the excess emission strength, as presently estimated, then a mean baryonic charge near the lower end of the allowed size range is preferred when standard dark matter distributions are assumed.

One may also hope to find a dark matter signal in mass distributions of low density but large physical extent. This technique corresponds to maximizing the radial distance over which the line of sight integral in Eq. (2) extends. The most important example of this type of observational constraint comes from observations of the all sky isotropic background. As the dark matter is known to have been present since well before the time of CMB formation any observational signature imprinted on the isotropic background will have been developing since that time. The possibility of a nugget contribution to the cosmic radio background has been considered [9] and was found to be consistent with observations. In fact, such a contribution may help to explain the unexpected rise in sky temperature at long wave lengths as observed by ARCADE2.

Demanding that the nugget contribution to the galactic and cosmological backgrounds not exceed the observed limits requires that the average baryon number is greater than $10^{25}$. If the nuggets are to provide the observed excesses in diffuse emission mentioned above a baryon number just slightly above this value is favored over the heavier end of the theoretically allowed range.

Indirect detection techniques may provide both general constrains and possible hints as to the nature of the dark matter, but they are also limited in their scope. The contribution to the various diffuse backgrounds from know astrophysical sources remains uncertain, and some of the observed excesses may require a modification to our understanding of astrophysical processes rather than the inclusion of a new contribution from dark matter. It is also difficult to translate any potential indirect dark matter signal into a specific model of its fundamental nature as many different dark matter candidates may be constructed in order to explain a particular signature. While observations and background modeling are steadily improving these challenges are unlikely to be overcome in the near future. As such these observations are strongly complimented by a parallel direct search program.

\subsection{Direct detection and ground based searches}

Conventional dark matter searches primarily target weakly interacting massive particle (WIMP) type dark matter. These searches assume a relatively large particle flux but a small interaction cross-section so that the emphasis is on detector sensitivity. For the type of high mass dark matter candidate considered here, detection is primarily limited by a detector's sensitivity to very low flux events. As such, most conventional dark matter searches have too small a detector area to place strong constraints on the abundance of quark nuggets. Assuming a standard dark matter profile the flux of nuggets through the earth's surface may be estimated as,

$$
\Phi_{N} \approx \frac{\rho_{D M} v_{D M}}{M_{N}} \approx \frac{10^{24}}{B_{N}} \mathrm{~km}^{-2} \mathrm{yr}^{-1}
$$

which is considerably below the sensitivity of any current dark matter search program. As the nuggets are strongly interacting, and have a macroscopically large cross section, the sensitivity of a given experiment to the presence of quark nugget dark matter scales directly with detector area and total run time.

The passage of a quark nugget through a detector will be a complex process of elastic scattering and thermalization. This does not easily lend itself to a general discussion and would require a detector by detector analysis. Fortunately, the majority of nuggets in our scenario are composed of antiquarks, whose interactions with the surrounding matter are dominated by annihilation to photons. The partial thermalization of annihilation energy within the nugget extends the bright photon spectrum from the gamma-ray down to the microwave. As such, most detectors with a photon count based trigger will be sensitive to the passage of an antiquark nugget.

Neutrino detectors such as Super-K and SNO have cross sectional areas on the order of hundreds of square meters and run times of up to the decade scale. As such, their non-detection of the intense photon burst that would accompany an antiquark nugget passing through the detector implies a minimum nugget baryon number of $B_{M I N} \sim 10^{22}$. The strongest present limits based on ground based detectors are likely those imposed by the IceCube monopole search program. The IceCube result implies $B>10^{25}[10]$.

A somewhat weaker constraint may also be obtained by considering a potential nugget contribution to the geothermal energy budget of the earth. Requiring that the nugget contribution to the heating of the earth's interior not exceed the present uncertainty in the total geothermal energy budget implies $B>10^{25}$ [11].

\section{Constraints from cosmic ray detectors}

While the flux of nuggets implied by the present bounds on their baryon charge, as expressed in Eq. (3), is small by the standards of conventional dark matter searches it is comparable to the flux of ultrahigh energy cosmic rays. As such it is well worth asking whether cosmic ray observatories may also be sensitive to dark matter candidates of this type. To this end I will develop a phenomenological description of the passage of a quark nugget through the earth's atmosphere, highlighting those properties most relevant to cosmic ray detectors.

\subsection{Basic considerations}

Dark matter nuggets will carry typical galactic velocities distributed around the $v \sim 200 \mathrm{~km} / \mathrm{s}$ scale. A nugget of matter striking the earth will elastically scatter a small column of atmospheric molecules. The small cross section to mass ratio of the nugget means that its velocity remain 
essentially unchanged during this process (in fact only nuggets at the lowest end of the allowed mass range will be significantly slowed as they pass through the entire earth). These events will deposit kinetic energy in the atmosphere and the earth's surface, but only in a localized way that is unlikely to produce a clear signal in large scale cosmic ray detectors. The same momentum considerations apply to nuggets of antiquarks, but in this case atmospheric molecules striking the nugget are likely to annihilate rather than simply scattering elastically. There is, consequently, significantly more energy released in the later case and much of it is released in the more readily detectable form of high energy photons and relativistic particles. In the case of a large nugget the total energy produced may be as much as $10^{7} \mathrm{~J}$. This is considerable more energy than is deposited in an air shower initiated by a conventional high energy cosmic ray, so these events may well be detectable, provided this energy is partially released in channels to which cosmic ray detectors are sensitive. In what follows I will focus on the more energetic class of events in which the nugget crossing the atmosphere is composed of antiquarks. Much of this discussion will be based on results and estimates that follow from considerations first introduced earlier introductory work [12].

An atmospheric molecule striking the nugget will first pass through the electrosphere shedding its electrons, which annihilate to produce gamma rays, roughly half of which will be emitted as ionizing radiation into the surrounding atmosphere. The nuclei will then strike the quark matter core and annihilate, generally to a pair of back to back jets. The majority of the energy released in nuclear annihilations will be thermalized within the nugget and subsequently emitted as thermal radiation. The thermal emission spectrum of the nuggets will extend from the microwave possibly up to $\mathrm{x}$-ray energies as the nugget is heated by increasing numbers of annihilations. Finally, in the case where a nuclear annihilation happens very near the surface of the nugget and one of the jets is directed back towards the surface particle emission may also occur. The primary annihilation products will be strongly interacting particles which will remain bound to the nugget, however these may decay to leptons which are not strongly bound to the quark matter. The electrons and positrons are not able to penetrate the electrosphere, but relativistic muons are, and these will dominate charged particle emission from the nugget. While this complicated production chain makes muon emission a relatively rare occurrence muons can travel a relatively long distance within the atmosphere and will be important in considering the possible detection of a quark nugget. From this basic microscopic picture of the interaction of a quark nugget with the surrounding atmospheric molecules we can estimate the larger scale consequences of a quark nugget's passage through the earth's atmosphere.

\subsection{Air shower development}

As the nugget crosses the atmosphere it will emit charged particles and ionizing radiation, initiating an air shower whose basic components are the same as those associated with an air shower initiated by an ultrahigh energy cosmic ray. The main difference in the case of a nugget initiated air shower is that the nugget itself remains intact while crossing the atmosphere. As such, the shower will develop over a much longer timescale associated with the galactic scale velocity of the quark nugget which travels roughly three orders of magnitude slower than an ultrarelativistic proton or nucleus. This introduces a much longer, millisecond, time scale into the process of shower development. As shower development is driven by the rate of annihilations it will scale with the atmospheric density rather than atmospheric depth, consequently these event will develop relatively close to the earth's surface. The high energy photons emitted from the nugget will produce an energetic core to the shower tightly centred on the nugget while the lower energy thermal photons and relativistic muons produce a larger scale, more diffuse, component of the shower.

\subsection{Fluorescence detection}

Fluorescence detection is complicated by the extended duration of the air shower. The production of visible and ultraviolet emission, to which fluorescence detectors are sensitive, will continue over time scales of a few milliseconds, making these events difficult to distinguish from a number of backgrounds including lighting and meteors. When the nugget is high in the atmosphere the fluorescence yield is dominated by photons produced by the emitted muons exciting atmospheric nitrogen just as it is in a conventional air shower. The number of muons moving through the atmosphere at a given time is estimated to be comparable to those in a conventional cosmic ray shower, however the muon production rate is subject to large uncertainties related to the motion of the hadronic jets through the quark matter. Very deep in the atmosphere the nugget will be hot enough that thermal emission dominates over atmospheric fluorescence. However, this effect is limited to so near the earth's surface that it would be difficult to directly observe. While the UV intensity generated by a nugget passing through the atmosphere is likely to be at a level that is, in principle, detectable by fluorescence detectors the long duration of these events make them difficult targets for such detectors and is likely to make any such observation at least partially ambiguous unless it is accompanied by a related surface based detection.

\subsection{Surface detection}

The production of muons in annihilations will continue all the way to the earth's surface. As they are produced in the annihilation of nuclear matter the resulting muons will be produced at typical QCD energies up to about a $\mathrm{GeV}$. Muons with this energy have a characteristic decay length of a few kilometers. This is important as it is greater than the typical spacing of surface detectors in cosmic ray detectors so that adjacent detectors may record near simultaneous muon hits, as required for the detector to trigger. However, as with fluorescence detection, the relatively slow velocity of the nugget complicates this picture. The charged particles in a conventional air shower are produced in a cascade with all particles traveling at 
essentially the speed of light, as such they arrive at the surface in a tight cluster over timescales of microseconds. Conversely, while the muons generated by a quark nugget initiated air shower will arrive at multiple detectors simultaneously, they will continue to arrive over the time between when the nugget first comes within a muon decay length of the earth's surface and the moment it actually hits the ground. As cosmic ray observatories become better able to correlate multiple detector strikes over millisecond durations this detection technique is likely to impose the strongest constraints on the flux of quark nuggets. The recent detection of millisecond scale bursts, associated with lightning strikes, has demonstrated the ability of both Telescope Array and the Pierre Auger Observatory surface detectors to observe this type of extended duration muon production even. If such an event were to be observed without an associated lightning strike it could possible be interpreted as a quark nugget induced air shower.

\subsection{Radio detection}

A quark nugget passing through the atmosphere will also produce a radio band signal which should be readably detectable by radio arrays intended for the detection of cosmic rays [13]. As in the case of fluorescence emission the radio band emission of a quark nugget crossing the atmosphere will be divided between thermal emission in the radio band and emission associated with the motion of the emitted relativistic muons through the atmosphere. Relativistic muons produced by the nugget are preferentially emitted in the forward direction, and are subsequently deflected by the earth's magnetic field. This process will give rise to geosynchrotron emission just as it does in a conventional air shower. The resulting radio signal will be at a similar intensity to that produced by a conventional air shower, but again with a significantly longer timescale. However, unlike the fluorescence signal, there is relatively little background for this type of millisecond duration radio burst. There will also be a component of the nugget's radio band emission associated with thermal emission. As with fluorescence emission, this will peak very close to the earth's surface. In this context the ANITA experiment is a very important detector platform. Currently available ANITA data produces constraints at a level comparable to the IceCube monopole observations and the next round of analysis is likely to provide even more stringent constraints [11].

\section{Conclusions}

There are broad classes of dark matter models outside the WIMP paradigm for which conventional dark matter searches do not provide strong constraints. In the case of high mass and low density dark matter the strongest constraints will come from detectors with the largest possible collection area. Cosmic ray detectors are able to seriously constrain this low flux parameter space, provided they are capable of recognizing a possible dark matter signal in their collected data. I have highlighted a specific dark matter model which will produce a relatively strong signal in most cosmic ray observatories, but most importantly for this or other models is the sensitivity of these detectors to longer duration events. As the dark matter velocity cannot surpass the escape velocity of the galaxy, any heavy dark matter candidate will have an atmospheric crossing time on the millisecond scale. The ANITA results, the IceCube monopole search and the detection of lightning related muon bursts at Auger and TA have demonstrated that cosmic ray detectors are capable of observing this type of long duration event. If this low flux limit continues to be pushed it will provide an entirely new set of constraints on the nature of dark matter which will strongly compliment current dark matter searches targeting a very different parameter space.

\section{References}

[1] A. R. Zhitnitsky, JCAP 0310, 010 (2003)

[2] D. H. Oaknin and A. Zhitnitsky, Phys. Rev. D 71, 023519 (2005)

[3] Witten, E., Phys. Rev. D 30, 272-285 (1984)

[4] D. H. Oaknin and A. R. Zhitnitsky, Phys. Rev. Lett. 94, 101301 (2005)

[5] K. Lawson and A. R. Zhitnitsky, JCAP 0801, 022 (2008)

[6] M. M. Forbes, K. Lawson and A. R. Zhitnitsky, Phys. Rev. D 82, 083510 (2010)

[7] M. M. Forbes and A. R. Zhitnitsky, JCAP 0801, 023 (2008)

[8] M. M. Forbes and A. R. Zhitnitsky, Phys. Rev. D 78, 083505 (2008)

[9] K. Lawson and A. R. Zhitnitsky, Phys. Lett. B 724, 17 (2013)

[10] Aartsen, M. G. and others Eur. Phys. J. C74, 2938 (2014)

[11] P.W. Gorham, Phys. Rev. D 86, 123005 (2012)

[12] K. Lawson, Phys. Rev. D 83, 103520 (2011)

[13] K. Lawson (2012), arXiv:1208.0042 